\title{
Relationship between physiological status, cognition, and age in adult men
}

\author{
STUART I. OFFENBACH \\ Purdue University, West Lafayette, Indiana \\ WOJTEK J. CHODZKO-ZAJKO \\ University of Alabama, Tuscaloosa, Alabama \\ and \\ ROBERT L. RINGEL \\ Purdue University, West Lafayette, Indiana
}

\begin{abstract}
In this study, we examined whether the slowing of responses that has been observed with increasing age is associated with physiological status. Adults differing on physiological status were administered a choice reaction time task with difficult and easy trials. The results indicated that choice reaction time was related to physiological status in the following manner: on difficult choice reaction time trials, high-fit adults responded faster than did low-fit adults. The present results suggested that low levels of physiological fitness may be associated with behavioral slowing on tasks requiring speeded responses and that the contribution of physiological factors to overall cognitive functioning might be more limited than has previously been thought.
\end{abstract}

One of the consistent findings in the study of aging has been that response speed decreases with age (Birren, Woods, \& Williams, 1980; Cerella, 1985; Salthouse, 1985). Salthouse (1985) and Birren et al. (1980) relate this finding to a slowing of fundamental neural events with increasing age. The result of this slowing is that older individuals take longer to complete cognitive processing. However, other factors-physical fitness and physiological status variables such as diseases that "result in local cell loss, interference with circulation, and ischemia" (Birren et al., 1980, p. 301)-may also be components of the response slowing that has been attributed to age (see Botwinick \& Storandt, 1974; Light, 1978).

Physiological status or fitness of otherwise healthy individuals is another factor that may be related to slowed reaction times (Baylor \& Spirduso, 1988; Spirduso, 1980). Young, but not old, adults who participate regularly in exercise programs have faster reaction times than those who do not participate (Botwinick \& Storandt, 1974). Spirduso (1975), on the other hand, found older (aged approximately 56 years), but not younger (aged about 24 years), "active sportsmen" had faster reaction times than nonactive older men. The absence of a clear relationship between physiological status and reaction time may reflect methodological differences in the assessment of either variable. Both Botwinick and Spirduso (like many other investigators) used subjective reports of participation in exercise as the measure of fitness and status in-

Requests for reprints should be addressed to Stuart I. Offenbach, Department of Psychological Sciences, Purdue University, West Lafayette, IN 47907. stead of direct physiological measures. Self-report measures make comparisons of the results from different studies difficult, because, among other factors, investigators often use varied criteria to define exercise.

There are more direct measures of physical status (using aerobic capacity or cardiovascular indicators), which, although rarely assessed, can serve as independent variables representing physiological status or fitness (Spirduso, 1980). For example, Tredway (1978) reported that oxygen uptake $\left(\mathrm{VO}_{2} \mathrm{max}\right)$ and reaction time among older adults were positively correlated. Woods (1981) compared the reaction times of high and low physical status groups (based on time using a stationary exercise bicycle, heartrate response patterns, and subjects' stated levels of physical activity). Woods' older fit subjects' reaction times were more similar to those of younger, less fit adults than their older, but less fit, cohorts. These results suggest that high physiological status may retard the "normal" agerelated slowing of responses.

That relationship was examined in more detail in the present study of choice reaction time. Physiological status was assessed, using Chodzko-Zajko and Ringel's (1987) index of physiological status (IPS), which combines several measures of diverse anthropometric, pulmonary, hemodynamic, and biochemical variables, all of them readily observable in virtually everyone in the elderly population, into a single score. As a result, the IPS is not heavily weighted or biased by any single process.

\section{METHOD}

\section{Subjects}

The 78 participants in this study were recruited from residential facilities and senior citizens' organizations. They had participated in a 
Table 1

Means and Standard Deviations for the Seven IPS Variables

\begin{tabular}{lrr}
\hline \multicolumn{1}{c}{ IPS Variable } & \multicolumn{1}{c}{$M$} & \multicolumn{1}{c}{$S D$} \\
\hline Systolic pressure & 134.6 & 14.03 \\
Diastolic pressure & 80.5 & 8.58 \\
Lean body weight & 67.6 & 7.94 \\
Forced vital capacity & 3.9 & 0.86 \\
Forced expiratory capacity & 3.1 & 0.75 \\
Serum triglyceride & 122.2 & 58.98 \\
Serum total cholesterol/ & 5.0 & 1.31 \\
High-density lipoprotein & & \\
\hline
\end{tabular}

larger study of the influence of physiological and biochemical factors on sensory and motor performance in the elderly (Chodzko-Zajko \& Ringel, 1987). As a result of failures of the apparatus, only 64 of the 78 participants completed the task. None of the participants had obvious visual impairments that interfered with the experimental task (e.g., they easily identified the task's large color stimuli). All of the subjects were male, and the mean age was 61 years $(S D=12$ years $)$.

\section{Procedures}

Evaluation of physiological status. The physiological and biochemical data were collected in two sessions at the Purdue University Human Performance Laboratory. The IPS included the following variables: lean body weight, systolic blood pressure, diastolic blood pressure, forced vital capacity, forced expiratory volume, serum triglyceride, and serum total cholesterol/high-density lipoprotein. The "fasting"' blood sample for the biochemical analyses was collected on a different day from the physiological testing session.

A factor analysis was computed among the physiological variables (principal axis solution and verimax rotation procedures were used; see Chodzko-Zajko \& Ringel, 1987, for a more detailed description of the results of this analysis). There were three factors in the physiological data: pulmonary function, blood lipids and body weight, and blood pressure. The IPS score was a combination of the three factor scores, each weighted by its respective eigen value. The resulting IPS scores were significantly correlated with age $(r=-.53, p<.01)$, suggesting that IPS declines with age (see Table 1 for the means and SDs for our subjects).

Choice reaction time task. The self-paced reaction time task consisted of $\mathbf{4 0}$ trials each (order randomized) of pairs of previously scaled very discriminable colors (red and yellow) and pairs that were less discriminable (blue and green). Stimulus selection was important, since age differences in either response speed or perceptual sensitivity could have affected choice reaction time. The task stimuli were produced by passing light from 24-V tungsten filament bulbs through Kodak Wratten filters. The stimuli were displayed in a box that had three $7.6 \times 10.2 \mathrm{~cm}$ windows in a row (14 cm apart, center to center) and subtended a visual angle $5.4^{\circ} \times 7.3^{\circ}$. The angle between stimulus windows, edge to edge, was $10^{\circ}$. On each trial, two colored squares were displayed in the center window, one in the top half of the window, the other in the bottom half. The subject then pressed the left or right window panel (always using the same finger), depending on which color square was in the top position of the center window. In order to exclude memory and minimize learning as factors in performance, the left and right windows were labeled appropriately so that the subject could see which panel to press. The instructions emphasized both speed and accuracy.

\section{RESULTS}

Prior to analysis of the reaction time results, subjects were assigned to subgroups on the basis of IPS scores (high vs. low) and age (old vs. middle-aged). The mean age of the two high and low IPS groups and the IPS scores for the two age levels in these post hoc subgroups were not significantly different $\left[F_{\text {IPS }}(1,65)=1.28\right.$, $F_{\text {Age }}(1,65)=2.71$, both $p s>.05$; see Table 2].

\section{Latency Analyses}

The first analysis was a subjects $\times$ trials repeatedmeasures analysis of variance of latencies (averaged over four blocks of 20 trials). The latencies did not change over trials $[F(3,186)=2.21, p>.05]$. Next, correlations were computed for the latency measures with IPS and with age. As expected, age was positively correlated with overall latency $(r=.57)$, and IPS was negatively correlated with overall latency $(r=-.38)$. An unweighted means analysis of variance with two variables between subjects (age and IPS level from Table 2) and one variable within trials (easy red-yellow trials vs. difficult blue-green trials) was computed on the latency data. The main effects for IPS $[F(1,60)=7.09]$, age $[F(1,60)=14.47]$, and trial type variable $[F(1,60)=94.49]$ were significant (all $p \mathrm{~s}<.05)$. Latencies were longer for the low IPS subjects, the older adults, and on the difficult blue-green colors. The age $\times$ trial type interaction was the only significant interaction $[F(1,60)=8.57]$, and latencies increased more from the easy to the difficult trials for the older subjects $(+.13 \mathrm{sec})$ than for the middle-aged subjects $(+.07 \mathrm{sec}$; see Table 3$)$.

\section{Analyses of Responses}

A repeated-measures analysis of variance (identical to those described above) yielded a significant trial type main effect $[F(1,60)=111.24]$ and an IPS $\times$ trial type interaction $[F(1,60)=15.46]$. As expected, there were fewer correct responses on the difficult blue-green trials: 32.6 versus 37.8 . The interaction means indicated that highIPS adults made fewer correct responses on the difficult trials than on the easy trials ( 31.1 vs. 38.0). Low IPS subjects performed similarly on both kinds of trials (33.7 on blue-green trials and 36.8 on red-yellow trials).

\section{DISCUSSION}

Salthouse (1985) suggested that the slowing of responses with age might not be an indication of nervous system pathology but in fact may reflect normal developmental changes in speed of processing information. To the extent that the IPS estimates the integrity of the physiological sys-

Table 2

Mean Age and IPS Scores

\begin{tabular}{lcccc}
\multicolumn{5}{c}{ Mean Age and IPS Scores } \\
\hline Age Group & IPS Level & $N$ & $M_{\text {Age }}$ & IPS \\
\hline Young & High & 20 & 50.0 & 58.8 \\
& Low & 15 & 53.6 & 44.9 \\
Old & High & 11 & 71.3 & 58.8 \\
& Low & 23 & 73.6 & 41.5 \\
\hline
\end{tabular}

Table 3

Response Latencies for the Trial Type Interaction

\begin{tabular}{lccc}
\hline & \multicolumn{2}{c}{ Trial Type } & \\
\cline { 2 - 3 } \multicolumn{1}{c}{ Group } & Red-Yellow & Blue-Green & Total $M$ \\
\hline Middle Aged & $.64 \mathrm{sec}$ & $.71 \mathrm{sec}$ & $.68 \mathrm{sec}$ \\
Old & $.79 \mathrm{sec}$ & $.92 \mathrm{sec}$ & $.86 \mathrm{sec}$ \\
High IPS & $.64 \mathrm{sec}$ & $.73 \mathrm{sec}$ & $.68 \mathrm{sec}$ \\
Low IPS & $.77 \mathrm{sec}$ & $.88 \mathrm{sec}$ & $.83 \mathrm{sec}$ \\
Trial Means & $.70 \mathrm{sec}$ & $.79 \mathrm{sec}$ & \\
\hline
\end{tabular}


tems that contribute to changes in CNS timing, that suggestion was supported. Reaction time was negatively related to IPS scores, and accuracy tended to increase with IPS. Thus, adults whose score on the IPS was low responded more slowly and had more difficulty in responding correctly. Although it may never be possible to completely separate the effects of age and fitness (because of inherent correlations between the two), our results suggest that nonpathological physiological changes associated with general physiological status also contribute to what has been considered age-related slowing of behavior.

The validity of this conclusion depends in part on having an accurate estimate of physiological status, fitness, or health. Too often self-report data have substituted for more direct measures, probably because many older adults are unable to tolerate the procedures required for the assessment of many physiological variables. For example, one frequent measure of physical fitness, maximal oxygen consumption $\left(\mathrm{VO}_{2} \mathrm{max}\right)$, is typically measured either by testing subjects to the point of exhaustion or by extrapolating from shorter submaximal periods of exercise (e.g., treadmill walking). However, as many as $30 \%$ of elderly subjects over 60 years of age are unable to attain the "oxygen plateau" criterion required for "testing to exhaustion" (Sidney \& Shephard, 1977). As a result, the more infirm elderly may be excluded from the very studies designed to determine how their physiological status is related to behavior. Similarly, indirect estimation of $\mathrm{VO}_{2} \max$ from obtained submaximal data may underestimate direct measurements by as much as $25 \%$ among elderly subjects (Tonino \& Driscoll, 1988).

These and other factors may limit the generality of studies of aging and health-status effects. Often the subjects we are most interested in are excluded or unable to participate in our research. In the present experiment, having an accurate measure of physiological status enabled us to determine that the relationship between choice reaction times and differing physiological status was similar to that between performance and age on other, similar tasks. Less fit adults, like older adults in other studies, had a tendency to respond more slowly and be a little more accurate.

\section{REFERENCES}

Baylor, A. M., \& SPIRduso, W. W. (1988). Systematic aerobic exercise and components of reaction time in older women. Journals of Gerontology: Psychological Sciences, 43, P121-P126.
Birren, J. E., Woods, A. M., \& Williams, M. V. (1980). Behavioral slowing with age: Causes, organization, and consequences. In L. W. Poon (Ed.), Aging in the 1980s: Psychological issues (pp. 293-308). Washington, DC: American Psychological Association.

BotWINICK, J., \& STORANDT, M. (1974). Cardiovascular status, depressive affect, and other factors in reaction time. Journal of Gerontology, 29, 543-548.

Cerella, J. (1985). Information processing rates in the elderly. Psychological Bulletin, 98, 67-83.

Chodzko-Zajko, W. J., \& Ringel, R. L. (1987). Physical fitness measures and sensory and motor performance in aging. Experimental Gerontology, 22, 317-328.

LIGHT, K. C. (1978). Effects of mild cardiovascular and cerebrovascular disorders on serial reaction time performance. Experimental Aging Research, 4, 3-22.

SAlthouse, T. A. (1985). Speed of behavior and its implications for cognition. In J. E. Birren \& K. W. Schaie (Eds.), Handbook of the psychology of aging (2nd ed., pp. 400-426). New York: Von Nostrand Reinhold.

SidNEy, K. H., \& SHEPHARD, R. J. (1977). Maximum and submaximum exercise tests in men and women in the seventh, eighth, and ninth decades of life. Journal of Applied Physiology, 43, 280-287. SPIRDUSO, W. W. (1975). Reaction and movement time as a function of age and physical activity level. Journal of Gerontology, 30, 435-440.

SPIRDUSO, W. W. (1980). Physical fitness, aging, and psychomotor speed: A review. Journal of Gerontology, 35, 850-865.

Tonino, R. P., \& Driscoll, P. A. (1988). Reliability of maximal and submaximal parameters of treadmill testing for the measurement of physical training in older persons. Journals of Gerontology: Medical Sciences, 43, M101-M104.

TREDWAY, V. A. (1978). Mood and exercise in older adults. Unpublished doctoral dissertation, University of Southern California, Los Angeles.

Woods, A. (1981). Age differences in the effect of physical activity and postural changes on information processing speed. Unpublished doctoral dissertation, University of Southern California, Los Angeles.

(Manuscript received July 17, 1989.) 